\title{
The Effectiveness of Pulmonary Rehabilitation in Comparison Interstitial Lung Diseases and Idiopathic Pulmonary Fibrosis
}

Kerti M, Kelemen $\mathrm{K}$ and Varga $\mathrm{J}^{*}$

Department of Pulmonary Rehabilitation, National Koranyi Institute for Pulmonology, Budapest, Hungary

\begin{abstract}
Background: There is a question about the correlation between exercise tolerance and improvement of functional markers in interstitial lung disease (ILD). We sought to investigate the correlation between exercise capacity and other functional markers as an effect of pulmonary rehabilitation in ILD and idiopathic pulmonary fibrosis (IPF).

Materials and methods: 30 patients with ILD (FVC: $64 \pm 21 \%$ pred, age: $54 \pm 8$ years, BMI: $28 \pm 6 \mathrm{~kg} / \mathrm{m}^{2}$ ) and 23 patients with IPF (FVC: $55 \pm 21 \%$ pred, age: $45 \pm 9$ years, BMI: $27 \pm 5 \mathrm{~kg} / \mathrm{m}^{2}$ ) participated in this study. Patients performed breathing techniques, personalized exercise training 2-3 times per day by cycling and treadmill. 6 minutes walking distance (6MWD), lung function $(\triangle \mathrm{FVC})$, chest wall expansion (CWE), grip strength (GS), maximal inspiratory pressure (MIP), breath holding time (BHT) and Quality of Life (QoL) were measured.
\end{abstract}

Results: Rehabilitation resulted significant improvement in functional markers in ILD (6MWD, FVC, IVC, CWE, MIP, GS, CAT and mMRC, p<0.05). Significant improvement was detected in 6MWD, IVC, CWE, MIP, CAT and mMRC $(p<0.05)$ in IPF. Stronger correlation was observed between 6MWD and in FVC IPF: $R^{2}=0.296$ vs. ILD $\mathrm{R}^{2}=0.0009$. QoL showed the following correlation with $6 \mathrm{MWD}$ IPF $\mathrm{R}^{2}=0.2, \mathrm{ILD} \mathrm{R}^{2}=-0.16$.

Conclusion: Pulmonary rehabilitation can improve functional parameters in ILD and in IPF, but the tendency of the improvement was different IPF compared to ILD groups in FVC and IVC to 6MWD.

Keywords: Interstitial lung disease; Idiopathic pulmonary fibrosis; Pulmonary rehabilitation; Functional parameters; Quality of life

\section{Introduction}

Interstitial lung diseases (ILD) represent some of the most severe chronic afflictions in the field of pulmonology [1]. Despite years of study and medical innovation, survival rates and quality of life scores of ILD patients remain low with a comprehensive treatment yet to be found [1].

Current pharmaceutical therapy has displayed limited efficacy in reducing the side-effect profile of ILD, rendering physical therapy the mainstay of treatment for the disease [2]. Despite this, the technical aspects of physical therapy and the foundations of its biological effects in ILD patients remain poorly understood and under-investigated [2]. This signifies a glaring gap in medical knowledge, greatly limiting our ability to analyse the properties of current rehabilitation protocols and further optimise them [2].

This paper aims to bridge the aforementioned gap by analysing the alterations brought upon key functional markers in ILD patients as brought upon of pulmonary rehabilitation programs and subsequently comprehending their subtle relationships in hopes of contributing to the creation of a comprehensive model for their therapeutic mechanisms.

Alike the malady whose symptoms it aims to counteract, rehabilitation therapy displays a vast array of subtle systemic effects, each of which that greatly impact disease progression, severity and QoL changes. To account for as many of these parameters as optimally possible, a wide variety of functional markers were selected for analysis by this study. Topics of particular interest include chest kinematics, respiratory capacity, oxygen absorption, peripheral circulation, functions of respiratory musculature, lung mechanics and metabolic alterations as measured in our subjects before, during and after pulmonary rehabilitation.

\section{Methods}

30 patients with ILD (interstitial lung diseases excluding IPF)
(FVC: $79 \pm 18 \%$ pred, age: $54 \pm 8$ years, BMI: $28 \pm 6 \mathrm{~kg} / \mathrm{m}^{2}$ ) and 23 patients with idiopathic pulmonary fibrosis (IPF) (FVC: $62 \pm 16 \%$ pred, age: $45 \pm 9$ years, BMI: $27 \pm 5 \mathrm{~kg} / \mathrm{m}^{2}$ ) participated in this study (Table 1 ). All of the patients gave written consent to the study in the Department of Pulmonary Rehabilitation. It was an observational study, using the general management of the patient. The study was approved by the Ethical Committee of the National Koranyi Institute for Pulmonology, Budapest, Hungary with 25/2017 registration number and the study was registered at ISRCTN registry with ISRCTN13019180 ID.

Patients performed a complex pulmonary rehabilitation program with chest wall-stretching, controlled breathing techniques and training, and personalized exercise training 2-3 times for 20-30 minutes per day by cycling and treadmill for 4 weeks. 6 minutes walking distance (6MWD) [3], lung function [4], chest wall expansion (CWE) [5], grip strength [6], maximal inspiratory pressure (MIP) [7] and breath holding time (BHT) [8] were measured. The quality of life and dyspnoea were evaluated by CAT $[9,10]$ and mMRC [11].

The breathing exercises were performed on an open-air corridor to take advantage of the special microclimate of the Hospital [12]. The patients learned controlled breathing and stretching technics, chest and spine mobilization exercises and muscle strengthening technics. The personalized training was performed on exercise bicycles and treadmills with continuous or interval training [13]. The training set

*Corresponding author: Janos Varga, Department of Pulmonary Rehabilitation, National Koranyi Institute for Pulmonology, Budapest, Hungary, Tel: +3613913374 Fax: +3613913285; E-mail: varga@koranyi.hu

Received September 28, 2018; Accepted October 03, 2018; Published October 10, 2018

Citation: Kerti M, Kelemen K, Varga J (2018) The Effectiveness of Pulmonary Rehabilitation in Comparison Interstitial Lung Diseases and Idiopathic Pulmonary Fibrosis. J Pulm Respir Med 8: 475. doi: 10.4172/2161-105X.1000475

Copyright: $\odot 2018$ Kerti M, et al. This is an open-access article distributed under the terms of the Creative Commons Attribution License, which permits unrestricted use, distribution, and reproduction in any medium, provided the original author and source are credited. 
Citation: Kerti M, Kelemen K, Varga J (2018) The Effectiveness of Pulmonary Rehabilitation in Comparison Interstitial Lung Diseases and Idiopathic Pulmonary Fibrosis. J Pulm Respir Med 8: 475. doi: 10.4172/2161-105X.1000475

Page 2 of 5

\begin{tabular}{|c|c|c|c|}
\hline $\mathbf{n = 5 3}$ & ILD $\mathbf{n = 3 0}$ & IPF $\mathbf{n = 2 3}$ & Significance \\
\hline Age (years) & $54 \pm 8$ & $45 \pm 9$ & n.s. \\
\hline Male:Female & $17: 13$ & $12: 11$ & n.s. \\
\hline BMI (kg/m²) & $28 \pm 6$ & $27 \pm 5$ & n.s. \\
\hline FVC (\%pred) & $64 \pm 21$ & $54 \pm 21$ & n.s. \\
\hline Hypertension & $16(53 \%)$ & $13(56 \%)$ & n.s. \\
\hline Diabetes & $7(23 \%)$ & $7(30 \%)$ & n.s. \\
\hline Atherosclerosis & $8(27 \%)$ & $6(22 \%)$ & n.s. \\
\hline Pulmonary hypertension & $10(33 \%)$ & $8(35 \%)$ & n.s. \\
\hline Nintenadib therapy & 0 & $8(35 \%)$ & p<0.05 \\
\hline ILD: Interstitial lung disease, IPF: Idiopathic pulmonary fibrosis, BMI: Body \\
mass index, FEV1: Forced expiratory volume in the first second
\end{tabular}

Table 1: Patients`characteristics.

was according to the patients' requirements. The protocol was defined considering the stage of ILD, the actual status of the heart, comorbidities and blood gas value. At the beginning and at the end of the program the different functional parameters were measured:

\section{Lung function}

According to ATS/ERS guidelines, all patients underwent pulmonary function testing $\left(\mathrm{V}_{\max } 229\right.$ and Autobox 6200, Sensormedics) including spirometry, body pletysmography and diffusion capacity measurements [4].

\section{Six-minute walking distance (6MWD)-test}

It was measured on the corridor along of 33 meters line $[3,12]$. The patients were asked to walk as fast as possible. The oxygen saturation, the heart rate and the modified Borg scale-scores were evaluated $[3,12]$. The protocol was standardized based on international guideline.

\section{Chest wall expansion (CWE) measurement}

Chest wall expansion measurement was based on the difference of chest circumferences at deep inspiration and expiration. It was measured at the level of processus xyphoideus $[5,14,15]$.

\section{Grip strength (GS)}

Kern hand grip dynamometer (2016 KERN \& SOHN GmbH | Ziegelei 1 | 72336 Balingen - Germany) was used to identify the peripherial muscles force [6].

\section{Maximal inspiratory pressure (MIP)}

We used a special digital instrument, Power Breathe K1 (POWERbreathe International Limited) to evaluate MIP. We needed the patient's height, weight, age, sex to calculate the degree of diaphragm force (very poor, poor, average, fair, good, very good). Patients were asked to exhale maximally than to take the $\mathrm{K} 1$ into the mouth with a sudden, maximal inspiration [7].

\section{Breath holding time (BHT)}

It is measured after a maximal exhalation and then a maximal inhalation. The subjects have to hold the air inside as long as possible with closed noses and mouth [8].

\section{Quality of life (QoL) marker}

It was assessed by CAT questionnaire. We could get information about different symptoms of these diseases (coughing, sputum, breathlessness, physical activity level) $[9,10]$. We also measured the dyspnea degree of the patient with mMRC [11].

\section{Statistical analysis}

Patient characteristics, lung function, resting and exercise functional variables were compared by a paired t-test, a nonparametric sign test, and a Wilcoxon signed-rank test. Significance was accepted at $\mathrm{p}<0.05$ level. The distribution around the mean was expressed as \pm SD in tables. Scatterplot distribution was observed in Figures 1-4. Distributions were tested for normality by the Kolmogorov-Smirnov

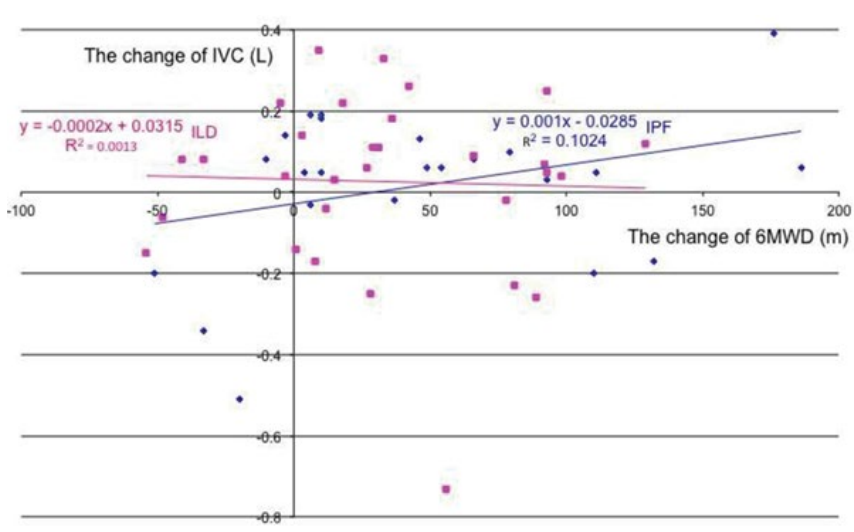

Figure 1: Correlation between the change of IVC $(L)$ and 6MWD $(m)$ as an effectiveness of the pulmonary rehabilitation in the ILD and IPF groups (IVC: Inspiratory vital capacity, 6MWD: Six/minute walking distance, ILD: Interstitial lung disease, IPF: Idiopathic pulmonary fibrosis).

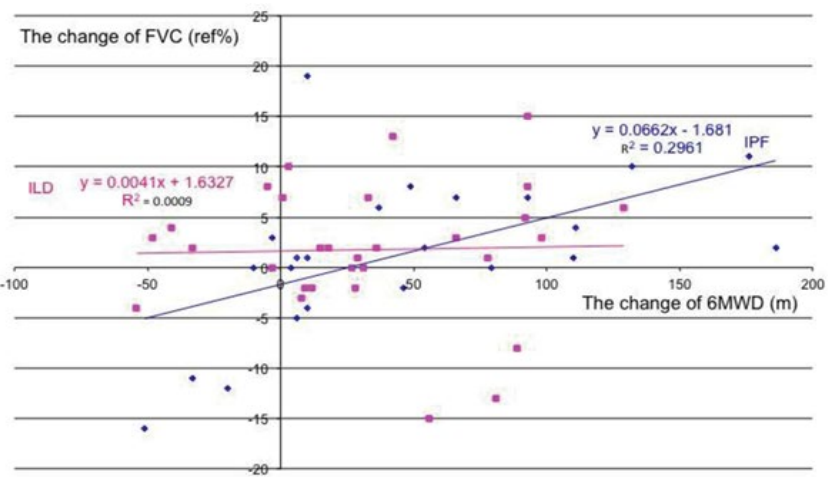

Figure 2: Correlation between the change of FVC (ref\%) and 6MWD (m) as an effectiveness of the pulmonary rehabilitation in the ILD and IPF group (FVC: Forced vital capacity, 6MWD: Six/minute walking distance, ILD: Interstitial lung disease, IPF: Idiopathic pulmonary fibrosis).

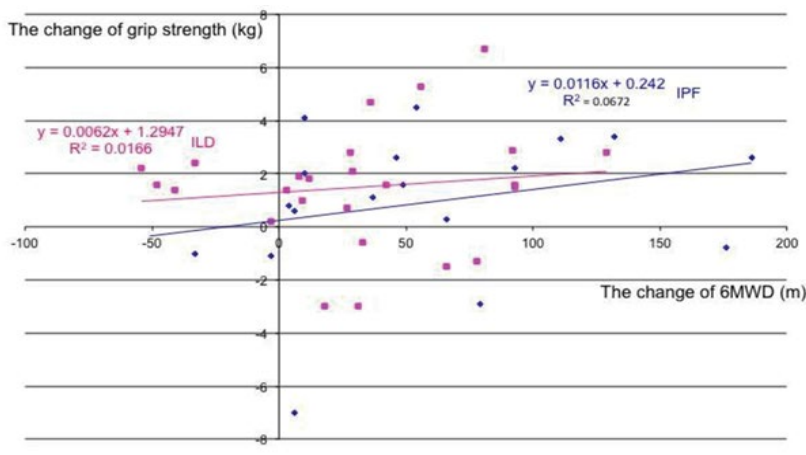

Figure 3: Correlation between the change of grip strength $(\mathrm{kg})$ and 6MWD (m) as an effectiveness of the pulmonary rehabilitation in the ILD and IPF group (6MWD: Six/minute walking distance, ILD: Interstitial lung disease, IPF: Idiopathic pulmonary fibrosis). 


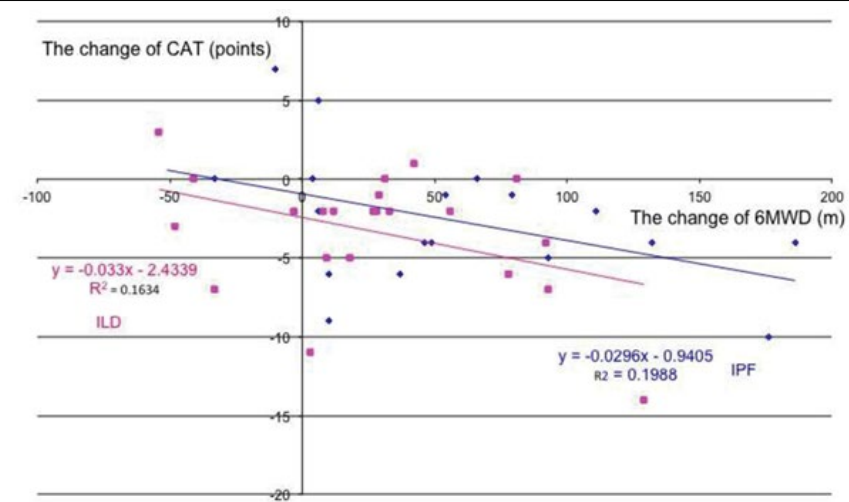

Figure 4: Correlation between the change of CAT (points) and 6MWD $(\mathrm{m})$ as an effectiveness of the pulmonary rehabilitation in the ILD and IPF group (CAT: COPD Assessment Test, 6MWD: Six/minute walking distance, ILD: Interstitial lung disease, IPF: Idiopathic pulmonary fibrosis).

test. Pearson correlation coefficient was calculated between the change in functional parameters and the health status markers.

\section{Results}

In general, pulmonary rehabilitation resulted improvement in exercise tolerance, chest hyperinflation, chest kinematics, respiratory and peripherial muscle function and quality of life (Table 2). We detected significant improvement in the IPF group in FVC\%pred, IVC\%pred, 6MWD, CWE and GS (Table 2). Significant improvement was detected in FVC\%pred, IVC\%pred, 6MWD, CWE in the ILD group (Table 2). We observed the different pattern in the two measured group in terms of correlation between functional and quality of life markers. We detected a stronger correlation between 6MWD and FVC in IPF-group, $\mathrm{R}^{2}=0.65$ versus in ILD-group $\mathrm{R}^{2}=0.0003$. Correlation between FEV1\% and 6MWD was stronger in IPF-group: $\mathrm{R}^{2}=0.296$ and indifferent in ILD-group $\mathrm{R}^{2}=0.0009$. The smallest difference between the two groups was in CAT questionnaire to $6 \mathrm{MWD}, \mathrm{R}^{2}=-0.2$ in IPFgroup and $\mathrm{R}^{2}=-0.16$ in ILD-group.

\section{Discussion}

Pulmonary rehabilitation resulted improvement in exercise capacity, lung function, lung mechanics, chest kinematics, respiratory and peripherial muscle function and health status marker and dyspnoea questionnaire in interstitial lung diseases. Physiologically different pattern as a tendency was developed in the ILD and IPF groups in IVC and FVC to $6 \mathrm{MWD}$ as an effectiveness of pulmonary rehabilitation, but similar tendency was detected in grip strength and CAT to 6MWD.

IPF is a progressive chronic pulmonary disease, which is characterized by progressive shortness of breath and reduction in exercise tolerance [16]. Median survival rates are still remained less than five years from the time of diagnosis [16]. Medications can help IPF patients to live longer, but an important question is the quality of life of these patients. Which are the interventions to live better, to be more active; to experience less dyspnea, depression and anxiety; to achieve a greater sense of control over their disease. Pulmonary rehabilitation may give adequate answers for these questions [16]. We detected a worsen quality of life in IPF and pulmonary rehabilitation was effective in IPF

It is known, that quadriceps muscle dysfunction is an important factor to exercise intolerance in COPD, but there is few information in fibrotic idiopathic interstitial pneumonia (IIP) in this term. In this clinical study, the strength and endurance of quadriceps muscle to exercise capacity was investigated compared to patients with fibrotic IIP and healthy controls [17]. 25 patients with fibrotic IIP (FVC: 78.7 $\pm 14.0 \%$ pred, DLCO: $40.3 \pm 10.9 \%$ pred) and 33 age-matched healthy controls were compared. Quadriceps strength was assessed using magnetic femoral nerve stimulation (17). Patients were significantly weaker than controls based on quadriceps twitch force $(10.1 \pm 3.0 \mathrm{~kg}$ vs. $8.0 \pm 2.4 \mathrm{~kg} ; \mathrm{P}=0.013)$. Repetitive magnetic stimulation could not maintain quadriceps force in patients during the endurance protocol. In controls, there was a significant relationship between 6-min walk distance and quadriceps twitch force, and quadriceps endurance [17]. As a conclusion, quadriceps strength and endurance are reduced in patients with fibrotic IIP compared with healthy controls, but the correlation with exercise tolerance was weak [17]. We did not measure the quadriceps force, but as general peripherial and respiratory muscle strength was reduced in this population as we determined.

A clinical study was conducted to determine the effects of chronic corticosteroid administration on skeletal muscle strength, exercise capacity, activities of daily living (ADL) and health status in ILD patients. Forty-seven ILD patients treated with corticosteroids and 51 Medical Research Council dyspnea grade-matched ILD patients not treated with corticosteroids were investigated by isometric quadriceps muscle force (QF) and handgrip force (HF), pulmonary function, 6-min walk distance, ADL score and health status (Medical Outcomes Study 36-Item Short-Form Health Survey) [18]. QF and HF were significantly lower in subjects on corticosteroids than in the control patients. It need to underline that there were no significant differences in the 6MWD, ADL score and all subscales of the SF-36 between the groups. Inverse correlations were found between skeletal muscle strength and total amount of corticosteroids administered [18]. We also observed reduced respiratory and peripherial muscle force in our ILD population, but our ILD group was heterogenous based on corticosteroid treatment.

Morino et al. investigated that whether pulmonary, physical, and mental functions; dyspnea; and daily physical activity (PA) have influence on the 6MWD in IPF patients [19]. 38 outpatients with IPF were investigated. The $6 \mathrm{MWD}$ was correlated with age, baseline dyspnea index, vital capacity (VC), diffusion capacity of carbon monoxide, quadriceps force $(\mathrm{QF})$, dyspnea during the 6-minute walk test, and PA. Stepwise multiple regression analysis revealed that VC $(\beta=0.382), \mathrm{QF}$ ( $\beta=0.272)$, and PA $(\beta=0.574)$ were contributing factors of the $6 \mathrm{MWD}$ [19]. In patients with IPF, PA has a greater effect on the 6MWD than VC and QF. This result supports that the evaluation of daily PA, in addition to physiological and muscle functions, is important in patients with IPF [19]. Based on these observations we performed step-wise analysis between 6MWD and different functional parameters.

A clinical study investigated the prognostic role of physical activity (PA) and exertional desaturation (ED) to mortality in IPF [20]. 34 IPF patients were asked to fill in International Physical Activity Questionnaire, and $\mathrm{SpO}_{2}$ was assessed pre to post 6-min walking test $\left(\Delta \mathrm{SpO}_{2}\right)$. There was a 40 months follow-up period [20]. They found a significant correlation between PA, ED and mortality in IPF patients. These results underlined the clinical importance of PA and ED assessments in IPF risk stratification, prognosis prediction, and in pulmonary rehabilitation, also. Based on these results 100-105 min/ week physical activity was associated with a reduced mortality risk and better survival in IPF [20]. We did not perform a longitudinal follow-up of the patient yet, but we plan to make this analysis in the future.

Focusing the importance of pulmonary rehabilitation in IPF a Cochrane review was conducted. The aim of the review was to investigate whether pulmonary rehabilitation in patients with ILD 
Citation: Kerti M, Kelemen K, Varga J (2018) The Effectiveness of Pulmonary Rehabilitation in Comparison Interstitial Lung Diseases and Idiopathic Pulmonary Fibrosis. J Pulm Respir Med 8: 475. doi: 10.4172/2161-105X.1000475

Page 4 of 5

\begin{tabular}{|c|c|c|c|c|c|c|}
\hline \multirow{2}{*}{ Variables } & \multicolumn{3}{|c|}{ ILD group } & \multicolumn{3}{|c|}{ IPF group } \\
\hline & Before rehabilitation & After rehabilitation & Significance (p) & Before rehabilitation & After rehabilitation & Significance (p) \\
\hline FEV1 (\%pred) & $62 \pm 22$ & $64 \pm 22$ & 0.25 & $56 \pm 22$ & $57 \pm 18$ & 0.77 \\
\hline FVC (\%pred) & $64 \pm 21$ & $66 \pm 21$ & 0.04 & $55 \pm 21$ & $56 \pm 19$ & 0.38 \\
\hline IVC (\%pred) & $62 \pm 21$ & $64 \pm 21$ & $0 . ; 04$ & $52 \pm 21$ & $54 \pm 19$ & 0.03 \\
\hline $\begin{array}{c}\text { Chest wall } \\
\text { expansion }(\mathrm{cm})\end{array}$ & $2.88 \pm 1.82$ & $3.95 \pm 2.07$ & $<0.0$ & $2.24 \pm 1.62$ & $3.11 \pm 1.73$ & 0.01 \\
\hline 6MWD (m) & $380 \pm 101$ & $413 \pm 98$ & $<0.01$ & $286 \pm 116$ & $333 \pm 136$ & $<0.01$ \\
\hline $\operatorname{MIP}\left(\mathrm{H}_{2} \mathrm{O} \mathrm{cm}\right)$ & $72 \pm 18$ & $90 \pm 21$ & $<0.01$ & $84 \pm 14$ & $92 \pm 15$ & 0.05 \\
\hline GS (kg) & $28.9 \pm 10.7$ & $30.4 \pm 11.4$ & $<0.01$ & $23 \pm 11$ & $24 \pm 10$ & 0.09 \\
\hline $\mathrm{BHT}(\mathrm{sec})$ & $23 \pm 12$ & $26 \pm 12$ & 0.08 & $15 \pm 8$ & $18 \pm 10$ & 0.08 \\
\hline CAT & $22 \pm 5$ & $15 \pm 6$ & $<0.01$ & $21 \pm 6$ & $16 \pm 6$ & $<0.01$ \\
\hline $\mathrm{mMRC}$ & $3.5 \pm 0.9$ & $2.7 \pm 1.0$ & 0.04 & $2.8 \pm 0.8$ & $2.2 \pm 0.6$ & 0.04 \\
\hline
\end{tabular}

IPF: Idiopathic pulmonary fibrosis, ILD: Interstitial lung disease, FEV1: Forced expiratory volume in the first second, FVC: Forced vital capacity, IVC: Inspiratory vital capacity, 6MWD: Six-minute walking distance, MIP: Maximal inspiratory pressure, GS: Grip strength, BHT: Breathe holding time, CAT: COPD Assesment Test, mMRC: Modified Medical Research Dyspnoea Scale

Table 2: The change of functional and quality of life markers as an effectiveness of pulmonary rehabilitation in the IPF and ILD groups.

has beneficial effects on exercise capacity, symptoms, quality of life and survival compared with no pulmonary rehabilitation in patients with ILD. A priori subgroup analyses were specified for participants with idiopathic pulmonary fibrosis (IPF) and participants with severe lung disease (low diffusing capacity or desaturation during exercise). No adverse effects of pulmonary rehabilitation were reported [20]. Pulmonary rehabilitation improved the six-minute walk distance with weighted mean difference (WMD) of 44.34 metres and improved oxygen consumption (VO2) peak with WMD of $1.24 \mathrm{~mL} / \mathrm{kg} / \mathrm{min}$. Improvements in six-minute walk distance and $\mathrm{VO} 2$ peak were also seen in the subgroup of participants with idiopathic pulmonary fibrosis (IPF) [21]. Dyspnoea and quality of life improved following pulmonary rehabilitation for all participants on a variety of measures and for the subgroup of people with IPF. As a conclusion for the metaanalysis, pulmonary rehabilitation seems to be safe for people with ILD [21]. Improvements in functional exercise capacity, dyspnoea and quality of life are seen immediately following pulmonary rehabilitation, with benefits also evident in IPF. It is needed to point out that relatively few data available for the effectiveness of interstitial lung disease and little evidence was available regarding longer-term effects of pulmonary rehabilitation [21]. Pulmonary rehabilitation was effective in 6MWD, IVC, CWE, MIP, CAT and mMRC in ILD and IPF in our program. We did not detect any severe adverse event in ILD and IPF, so pulmonary rehabilitation was safe in both group.

Although only a small number of studies have been published to date in the topic of effectiveness of pulmonary rehabilitation in IPF $[22,23]$. Most studies have found significant short-term improvements in functional exercise capacity, quality of life, and level of perceived dyspnea. Long-term improvements or maintenance strategies of $\mathrm{PR}$ in IPF patients have not been adequately investigated yet. However, physical training seems to be the major component of all $P R$ programs [22]. A specific multidisciplinary PR program is needed for IPF patients. Pulmonary rehabilitation can be a therapeutic option for patients with IPF with the new specific drugs like nintedanib and pirfenidone [22]. We had a few patients who were on nintenadib therapy, which is available not for a long time.

Idiopathic pulmonary fibrosis (IPF) is a chronic, devastating, interstitial lung disease, which is characterized by pulmonary restriction, dyspnea, hypoxemia, exercise intolerance and poor quality of life (QOL). In a randomized controlled study thirty-two IPF were participated in a ET group $(n=15)$, participating in a 12 -week, twiceweekly 60 -min supervised ET-based pulmonary rehabilitation program, or in a control group $(\mathrm{n}=17)$ [24]. Cardiopulmonary exercise test, 6-min walking distance (6MWD) test, 30 -second chair-stand test, pulmonary function tests, dyspnea and QOL were assessed at baseline and at the end of the 12-week intervention [23-25]. Significant differences were observed between the ET and the control groups $\triangle 6 \mathrm{MWD}, \Delta \mathrm{VO} 2$ peak, $\Delta$ work rate, $\Delta$ anaerobic threshold, and $\triangle \mathrm{FVC} \%$ predicted. Dyspnea, QOL and 30-second chair-stand were also improved significantly following the program. ET resulted improvement in exercise tolerance, functional capacity, pulmonary function, dyspnea and QOL in patients with IPF, suggesting a short-term treatment efficacy for clinical improvement, and should be considered the standard care for IPF [24]. We did not perform a cardiopulmonary exercise test in this study, but we plan to perform it in the future for analysis of physiological effect of the rehabilitation in ILD and IPF in more details.

Improvement of endurance time was the most responsive exercise measurement for evaluating PR efficacy in patients with idiopathic pulmonary fibrosis in many studies [25-30]. In a prospective observational study 53 subjects with idiopathic pulmonary fibrosis were enrolled. The PR group underwent a 10-week outpatient PR program. The control group was observed without any additional intervention, including PR [26]. Five exercise measurements (endurance time [ET], peak work rate, peak oxygen consumption [VO2 ], 6-min walk distance, and incremental shuttle walk distance) were compared to detect the effectiveness of the PR program. In each group, 24 subjects completed the 5 measurements at baseline and after 10 weeks. All of the measured parameters were significantly different between the groups after 10 weeks $(\mathrm{P}<0.05)$ [25]. In the $\mathrm{PR}$ group, ET showed the most striking improvement among the 5 measurements $(\mathrm{P}<0.05)$. We also use different measurements for the effectiveness of pulmonary rehabilitation program including endurance time, 6-minutes walking distance and peak work rate. We did not have an experience in an outpatient program in this disease, our program was based on inpatient condition.

Our study had some limitations. The pulmonary rehabilitation program was somewhat shorter than the programs for patients with COPD, but the frequency and intensity of the training was so high that the total amount of the work was comparable to longer programs. Psychological assessment was not part of the program, we plan to make a program for it in the future. We plan to observe the duration of the effectiveness of the pulmonary rehabilitation in a longitudinal study in patients with ILD in the future. 
Citation: Kerti M, Kelemen K, Varga J (2018) The Effectiveness of Pulmonary Rehabilitation in Comparison Interstitial Lung Diseases and Idiopathic Pulmonary Fibrosis. J Pulm Respir Med 8: 475. doi: 10.4172/2161-105X.1000475

\section{Conclusion}

Pulmonary rehabilitation resulted improvement in lung mechanics, chest kinematics, respiratory and peripherial muscle function, exercise tolerance and quality of life in patients with ILD in general. Physiologically different pattern as a tendency was developed in the ILD and IPF groups in IVC and FVC to 6MWD as an effectiveness of pulmonary rehabilitation, but similar tendency was detected in grip strength and CAT to 6MWD.

\section{Conflict of Interest}

None of the authors reported conflict of interest.

\section{References}

1. Bourke SJ (2006) Interstitial lung disease: Progress and problems. Postgrad Med J 82: 494-499.

2. Jo HE, Troy LK, Keir G, Chambers DC, Holland A, et al. (2017) Treatment of idiopathic pulmonary fibrosis in australia and new zealand: A position statement from the thoracic society of australia and new zealand and the lung foundation australia. Respirol 22: 1436-1458

3. Balke B (1963) A simple filed test for the assessment of physical fitness. Rep 63-6. Rep CivAeromed Res Inst US: 1-8.

4. Quanjer PH, Tammeling GJ, Cotes JE, Pedersen OF, Peslin R, et al. (2003) Lung volumes and forced ventilatoryflows. Report working party standardization of lung function tests, european community for steel and coal. Official statement of the european respiratory society. Eur Respir J 16: 5-40.

5. Debouche S, Pitance L, Robert A, Liistro G, Reychler G (2016) Reliability and reproducibility of chest wall expansion measurement in young healthy adults. J Manipulative Physiol Ther 39: 443-449.

6. An KN, Chao NY, Askew LJ (1980) Hand strength measurement instruments Arch Phys Med Rehabil 61: 366-368.

7. Neumeister W, Rasche K, Maas P, Monnerjahn C, Singh B, et al. (1996) Reproducibility of computer-assisted mouth occlusion pressure measurements. Med Klin 91: 73-75.

8. Mirsky IA, Lipman E, Grinker RR (1946) Breath holding time in anxiety states. Fed Proc 5: 74.

9. Jones PW, Tabberer M, Chen WH (2011) Creating scenarios of the impact of COPD and their relationship to COPD Assessment Test (CAT ${ }^{\mathrm{TM}}$ ) scores. BMC Pulm Med 11: 4

10. Jones PW, Agusti AG (2006) Outcomes and markers in the assessment of chronic obstructive pulmonary disease. Eur Respir J 27: 822-832.

11. Launois C, Barbe C, Bertin E, Nardi J, Perotin JM, et al. (2016) The modified medical research council scale for the assessment of dyspnea in daily living in obesity: A pilot study. BMC Pulm Med 12: 61

12. Kerti M, Balogh ZS, Halasz A, Kelemen K, Varga J (2016) COPD assessment for symptoms and functional condition in pulmonary rehabilitation. ERS Annual Congress 2016, Amsterdam: P2218.

13. Varga J, Porszasz J, Boda K, Casaburi R, Somfay A (2007) Supervised high intensity continuous and interval training vs. self-paced training in COPD. Respir Med 101: 2297-2304.
14. Kenyon CM, Cala SJ, Yan S, Aliverti A, Scano G, et al. (1997) Rib cage mechanics during quiet breathing and exercise in humans. J Appl Physiol 83 1242-1255.

15. Aliverti A, Cala SJ, Duranti R, Ferrigno G, Kenyon CM, et al. (1997) Human respiratory muscle actions and control during exercise. J Appl Physiol 83: 12561269.

16. Swigris JJ, Brown KK, Make BJ, Wamboldt FS (2008) Pulmonary rehabilitation in idiopathic pulmonary fibrosis: A call for continued investigation. Respir Med 102: $1675-1680$.

17. Mendoza L, Gogali A, Shrikrishna D, Cavada G, Kemp SV, et al. (2014) Quadriceps strength and endurance in fibrotic idiopathic interstitial pneumonia. Respirol 19: 138-143.

18. Hanada M, Sakamoto N, Ishimatsu Y, Kakugawa T, Obase Y, et al. (2016) Effect of long-term treatment with corticosteroids on skeletal muscle strength, functional exercise capacity and health status in patients with interstitial lung disease. Respirol 21: 1088-1093.

19. Morino A, Takahashi H, Chiba H, Ishiai S (2017) Daily physical activity affects exercise capacity in patients with idiopathic pulmonary fibrosis. J Phys Ther Sci 29: 1323-1328.

20. Vainshelboim B, Kramer MR, Izhakian S, Lima RM, Oliveira J (2016) Physical activity and exertional desaturation are associated with mortality in idiopathic pulmonary fibrosis. J Clin Med 5: E73.

21. Dowman L, Hill CJ, Holland AE (2014) Pulmonary rehabilitation for interstitial lung disease. Cochrane Database Syst Rev 6: CD006322.

22. Kenn K, Gloeckl R, Behr J (2013) Pulmonary rehabilitation in patients with idiopathic pulmonary fibrosis--a review. Respiration 86: 89-99.

23. Dowman LM, McDonald CF, Hill CJ, Lee AL, Barker K, et al. (2017) The evidence of benefits of exercise training in interstitial lung disease: A randomised controlled trial. Thorax 72: 610-619.

24. Vainshelboim B, Oliveira J, Yehoshua L, Weiss I, Fox BD, et al. (2014) Exercise training-based pulmonary rehabilitation program is clinically beneficial for idiopathic pulmonary fibrosis. Respiration 88: 378-388.

25. McNarry MA, Harrison NK, Withers T, Chinnappa N, Lewis MJ (2017) Pulmonary oxygen uptake and muscle deoxygenation kinetics during heavy intensity cycling exercise in patients with emphysema and idiopathic pulmonary fibrosis. BMC Pulm Med 17: 26.

26. Sharp C, McCabe M, Hussain MJ, Dodd JW, Lamb H, et al. (2017) Duration of benefit following completion of pulmonary rehabilitation in interstitial lung disease-an observational study. QJM 110: 17-22.

27. Arizono S, Taniguchi H, Sakamoto K, Kondoh Y, Kimura T, et al. (2014) Endurance time is the most responsive exercise measurement in idiopathic pulmonary fibrosis. Respir Care 59: 1108-1115.

28. Vainshelboim $B$ (2016) Exercise training in idiopathic pulmonary fibrosis: Is it of benefit?. Breathe 12: 130-138.

29. Strookappe B, Elfferich M, Swigris J, Verschoof A, Veschakelen J, et al. (2015) Benefits of physical training in patients with idiopathic or end-stage sarcoidosisrelated pulmonary fibrosis: A pilot study. Sarcoidosis Vasc Diffuse Lung Dis 32 : 43-52.

30. Jackson RM, Gómez-Marín OW, Ramos CF, Sol CM, Cohen MI, et al. (2014 Exercise limitation in IPF patients: A randomized trial of pulmonary rehabilitation. Lung 192: 367-376. 